\title{
Using ROC curves to test models of recognition memory: The relationship between presentation duration and slope
}

\author{
ELLIOT HIRSHMAN and MARK HOSTETTER \\ University of North Carolina, Chapel Hill, North Carolina
}

\begin{abstract}
Many theories of memory predict that the slope of the $z$-transformed receiver-operating characteristic (ROC) curve should decrease with increases in the memory strength of old items. While several prior studies have failed to demonstrate this effect, the results of two experiments demonstrate that increasing presentation duration can reduce the slope of the $z$-transformed ROC curve. These results raise questions about the generality of prior results and of dual-process theories designed to accommodate those results. We close by emphasizing that determining the experimental circumstances that affect whether or not the slope of the $z$-transformed ROC curve decreases will provide important constraints on theories of recognition memory.
\end{abstract}

At the most general level, theories of recognition memory (Gillund \& Shiffrin, 1984; Glanzer, Adams, Iverson, \& Kim, 1993; Hintzman, 1988; Jacoby, 1991; Murdock, 1982) describe how encoding and retrieval processes determine underlying measures of memory strength, with consequent effects on memory performance. While many empirical tests of these theories focus on predictions regarding the average strength of items, Ratcliff and his colleagues (Ratcliff, McKoon, \& Tindall, 1994; Ratcliff, Sheu, \& Gronlund, 1992) have emphasized that it is also fruitful to test predictions regarding the relative variance of "new" and "old" items' strengths. Specifically, they follow classical signal detection theory and its assumption of unidimensional memory strength (Green \& Swets, $1966)$ in using the slope of the $z$-transformed receiveroperating characteristic (ROC) curve as a measure of the relative variance of the new and old distributions. Thus, predictions regarding the value of the relative variance of new and old items' strength can be examined by analyzing the slope of the $z$-transformed ROC. Similarly, the intercept of the $z$-transformed ROC provides a measure of the memory strength of the old distribution.

Many recent studies (Glanzer \& Adams, 1990; Gronlund \& Elam, 1994; Ratcliff et al., 1994; Ratcliff et al., 1992; Yonelinas, 1994, 1997) have examined the relationship between the slope of the $z$-transformed ROC curve and the memory strength of the old distribution. This relationship is of interest because there is a broad range of theoretical models that make predictions about it (Gillund

This research was supported by Research Grant SBR-9420248 from the National Science Foundation to the first author. The authors thank Bob Greene and two anonymous reviewers for comments on an earlier version of this manuscript. Correspondence should be addressed to E. Hirshman, Department of Psychology, Davie Hall, CB \#3270, University of North Carolina, Chapel Hill, NC 27599-3270 (e-mail: ellioth@email.unc.edu).
\& Shiffrin, 1984; Glanzer et al., 1993; Hintzman, 1988; Murdock, 1982; Yonelinas, 1994). For example, both the search of associative memory model (Gillund \& Shiffrin, 1984) and the attention-likelihood theory (Glanzer et al., 1993) predict that the relative variance of the new and old strength distributions, and consequently the slope of the $z$ transformed ROC curve, should decrease as the strength of old items increases. Results of empirical studies testing these predictions have been mixed, with some studies demonstrating that the slope of the $z$-transformed ROC curve is approximately constant (e.g., Ratcliff et al., 1994, Experiment 3) and other studies demonstrating that it decreases (e.g., Glanzer \& Adams, 1990; Yonelinas, 1994, Experiments 1 and 2) as a function of old item strength.

Yonelinas (1994) has attempted to bring theoretical understanding to this complex pattern of results by pointing out that the findings can be categorized in two distinct ways. First, Yonelinas points out that a range of studies dealing with variables, such as study-test lag, word frequency, word concreteness, word decoding, and list length, tend to produce decreases in slopes with increases in overall performance. On the other hand, in a second type of study, manipulations of the variable of presentation duration tend to have no effect on slope as presentation duration increases. Yonelinas uses a dual-process perspective in which both recollection and familiarity influence performance to explain these findings. Yonelinas proposes that when familiarity is constant, and recollection increases across the levels of a variable, the slope of the $z$ transformed ROC curve should decrease. However, if both familiarity and recollection increase across the levels of an experimental variable, the slope of the $z$-transformed ROC curve should remain approximately constant. Thus, Yonelinas explains those findings in which the slope of the $z$-transformed ROC curve decreases with increases in performance by hypothesizing that familiarity remains rel- 
atively constant, whereas recollection increases in these cases. He explains the finding with the variable of presentation duration in which the slope of the $z$-transformed ROC curve remains approximately constant by claiming that both familiarity and recollection increase when presentation duration is increased.

The primary purpose of this study was to show that, under a broad range of circumstances, the slope of the $z$ transformed ROC curve decreases with increases in presentation duration and performance. This finding raises questions about the generality of prior findings using the variable of presentation duration and suggests that this variable may have effects similar to those of other variables, such as list length. In doing this, the present results raise questions about the explanatory utility of the above version of the dual process account of the $z$-transformed ROC curve.

In Experiment 1, we pursued this purpose by manipulating two variables. First, we manipulated presentation duration during study, presenting some items for $500 \mathrm{msec}$ and other items for $2 \mathrm{sec}$. We used a very rapid presentation duration (i.e., $500 \mathrm{msec}$ ) as our initial duration to provide the strongest opportunity for increases in familiarity, as well as recollection, to influence performance. The influence of these joint increases should, according to Yonelinas (1997), operate to keep the slope of the $z$ transformed ROC approximately constant. We then examined the slope of the $z$-transformed ROC curve in these two conditions as a measure of whether the relative variance of the new and old distributions decreases with increases in presentation duration. In prior studies, Ratcliff et al. (1992), Ratcliff et al. (1994, Experiments 4 and 5), and Yonelinas (1994, Experiment 3) found that increasing presentation duration had little effect on the slope of the $z$-transformed ROC; it was approximately constant at values around .80. However, as discussed above, there have been many inconsistencies in the prior literature. To give one specific example, Gronlund and Elam (1994, Experiment 2) and Yonelinas (1994, Experiments 1 and 2) demonstrated that increasing list length decreased the slope the of $z$-transformed ROC, whereas Gronlund and Elam (1994, Experiment 1) and Ratcliff et al. (1994, Experiment 3 ) found that increasing list length did not influence slope. Consequently, we thought it important to ascertain the generality of prior findings on the effects of presentation duration. To foreshadow, our concerns were warranted, and we will demonstrate below that there are many situations in which increasing presentation duration decreases the slope of the $z$-transformed ROC (see also Glanzer, Kim, Hilford, \& Adams, 1999).

Our second manipulation focused on the nature of the confidence rating judgment task. In one condition, we asked subjects to make an old-new decision and then give a 3-point confidence rating for all items judged "old." We refer to this as a high-criteria rating because the criteria used to make the 3-point rating for old items were high on the memory strength axis. At other times, we asked subjects to make an old-new decision and then give a 3-point confidence rating for all items judged "new." We refer to this as a low-criteria rating because the criteria used to make the 3-point rating for old items were low on the memory strength axis.

A third condition was a symmetrical 4-point rating in which subjects judged an item as old or new and then gave a 2-point confidence rating. The last condition was a symmetrical 6-point rating scale in which subjects judged an item as old or new and then gave a 3-point confidence rating. These four conditions allowed us to replicate our findings using both traditional and nontraditional confidence rating procedures.

\section{EXPERIMENT 1}

\section{Method}

Subjects. The subjects were 32 students in an introductory psychology class who participated in partial fulfillment of a research participation requirement.

Design. The experiment used a two-factor within-subjects design. Presentation duration ( $500 \mathrm{msec}$ vs. $2 \mathrm{sec}$ ) was manipulated within subjects, as was rating task (high criteria vs. low criteria vs. 4 symmetric vs. 6 symmetric).

Materials. The materials were 640 words from Thorndike and Lorge (1944). These words were divided into four sets of 160 items, with each set being presented as the study-test materials for one of four blocks. Two lists consisting of 80 items were constructed from each set of 160 items, with each list serving as either study or distractor items depending on the counterbalancing. Each of the 80item study lists were separated into two blocks of 40 items each. For each study list, each block of 40 items was assigned to one presentation duration condition for half of the subjects and to the other presentation duration condition for the remaining subjects. Similarly, each of the 80 -item study lists were presented at study for half of the subjects and served as distractor or new items for the other half of the subjects. Finally, each set of 160 items was rotated through the four rating task conditions for every group of 4 subjects. This counterbalancing ensured that (1) all items appeared equally often as old and new items, (2) all old items appeared equally often in each presentation duration, (3) all old and new items appeared equally often in each rating task conditions, and (4) serial position of items was equated across the levels of each experimental factor.

Procedure. The experiment consisted of four study-test periods in which each study list was blocked with its corresponding test list Prior to the first study list, the subjects were told they would be presented with items on a computer monitor. They were warned that some items would be presented rapidly. Following these brief instructions, 40 items were presented in one of the presentation duration conditions, and then the remaining 40 items were presented in the other presentation duration condition. Following this 80 -item study list, the subjects were asked to do 1 min of backward counting during the retention interval. After this brief retention interval, a recognition memory test presented the 80 study items and the corresponding 80 new items in random order. For each test item, the subjects were asked to make an old-new judgment, followed by a confidence rating. The nature of this confidence rating varied as a function of the rating task condition.

In the low-criteria condition, the subjects were asked to report their confidence for the items they had called "new" on a 3-point scale, with 1 representing low confidence and 3 representing high 


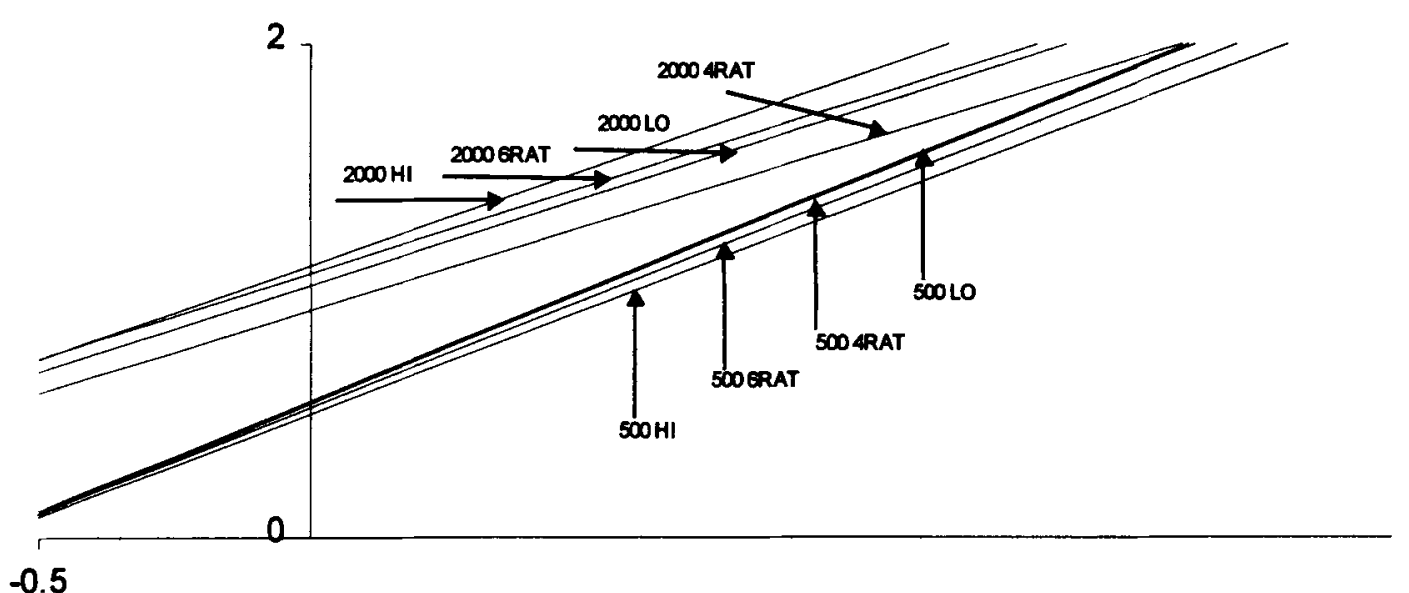

Figure 1, $z-\mathrm{ROC}$ as a function of presentation duration and rating task.

confidence. In the high-criteria condition, the subjects were asked to report their confidence for the items they had called "old" on a 3 -point scale, with 1 representing low confidence and 3 representing high confidence. In the 4-symmetric condition, the subjects were asked to report their confidence for all items on a 2-point scale, with 1 representing low confidence and 2 representing high confidence. In the 6-symmetric condition, the subjects were asked to report their confidence for all items on a 3-point scale, with 1 representing low confidence and 3 representing high confidence.

Following the first recognition memory test, the subjects proceeded to the next study-test block and continued until they had completed all four study-test blocks. Following this, they were debriefed and excused.

Data analysis. We used both linear regression and maximum likelihood estimation (Ogilvie \& Creelman, 1968), assuming variability in the 2 -transformations of both the hits and the false alarms, to estimate the slopes and intercepts of the $z$-transformed ROC. We then compared the estimated slopes and intercepts across conditions using analyses of variance (ANOVAs). The results with both estimation procedures were identical, and we present only the estimates from the maximum likelihood procedure for ease of exposition. In addition, because the slope of the $z$-transformed ROC may be forced to equal 1 when discriminability is 0 and this may artificially inflate slopes at fast presentation durations, we conducted our analyses both with and without subjects who demonstrated intercepts below .1 (i.e., those with very low levels of discriminability). Results were identical in both analyses, and we present only the complete analyses for ease of exposition.

\section{Results and Discussion}

The central result of Experiment 1 was the consistent finding that increasing presentation duration from $500 \mathrm{msec}$ to $2 \mathrm{sec}$ decreased the slope of the $z$-transformed ROC. This suggests that prior null effects of presentation duration on slope (e.g., Ratcliff et al., 1992) are not general. The mean slopes and intercepts are presented in Table 1 as a function of presentation duration and rating task. The $z$-ROCs incorporating these values are presented in Figure 1 as a function of presentation duration and rating task. Visual inspection of the figure and statistical analyses of the parameters of the $z$-ROCs of the individual subjects confirmed the preceding conclusion. A $2 \times 4$ within-subjects ANOVA on the intercepts produced one significant effect. Consistent with the common view that increasing presentation duration increases discriminability, intercepts increased with increased presentation duration $\left[F(1,31)=72.71, M S_{\mathrm{e}}=0.22, p<.001\right]$. A $2 \times 4$ within-subjects ANOVA on the slopes also produced one significant effect. Confirming that increasing presentation duration decreases relative variance, slopes decreased with increased presentation duration $\left[F(1,31)=47.20, M S_{\mathrm{e}}=\right.$ $0.03, p<.001]$. No other effects approached the traditional criterion for significance (all $p \mathrm{~s}>.10$ ).

A recent report by Glanzer et al. (1999) is consistent with the conclusions just drawn. Specifically, Glanzer et al. (1999) demonstrated (Experiments 1 and 2) that increasing presentation duration decreased the slope of the $z$-transformed ROC, a finding that buttresses the preceding claim that prior null effects of presentation duration are not general.

A qualm one might have about the results of Experiment 1 is that the higher slope in the $500-\mathrm{msec}$ presentation duration condition might have arisen as an artifact of the averaging of the subjects whose discriminability was

Table 1

Mean Slopes and Intercepts (and Their Standard Deviations) as a Function of Rating Task and Presentation Duration

\begin{tabular}{|c|c|c|c|c|}
\hline \multirow{2}{*}{$\begin{array}{c}\text { Presentation } \\
\text { Duration } \\
\end{array}$} & \multicolumn{2}{|c|}{ Intercept } & \multicolumn{2}{|c|}{ Slope } \\
\hline & $M$ & $S D$ & $M$ & $S D$ \\
\hline \multicolumn{5}{|c|}{ High-Criteria Rating } \\
\hline $\begin{array}{l}500 \mathrm{msec} \\
2 \mathrm{sec}\end{array}$ & $\begin{array}{l}0.50 \\
1.10\end{array}$ & $\begin{array}{l}0.36 \\
0.56\end{array}$ & $\begin{array}{l}0.83 \\
0.76\end{array}$ & $\begin{array}{l}0.27 \\
0.39\end{array}$ \\
\hline \multicolumn{5}{|c|}{ Low-Criteria Rating } \\
\hline $\begin{array}{l}500 \mathrm{msec} \\
2 \mathrm{sec}\end{array}$ & $\begin{array}{l}0.55 \\
1.02\end{array}$ & $\begin{array}{l}0.43 \\
0.46\end{array}$ & $\begin{array}{l}0.89 \\
0.70\end{array}$ & $\begin{array}{l}0.29 \\
0.30\end{array}$ \\
\hline \multicolumn{5}{|c|}{ Symmetrical 4-Point Rating } \\
\hline $\begin{array}{l}500 \mathrm{msec} \\
2 \mathrm{sec}\end{array}$ & $\begin{array}{l}0.54 \\
0.92\end{array}$ & $\begin{array}{l}0.32 \\
0.40\end{array}$ & $\begin{array}{l}0.87 \\
0.67\end{array}$ & $\begin{array}{l}0.21 \\
0.28\end{array}$ \\
\hline \multicolumn{5}{|c|}{ Symmetrical 6-Point Rating } \\
\hline $\begin{array}{l}500 \mathrm{msec} \\
2 \mathrm{sec}\end{array}$ & $\begin{array}{l}0.53 \\
0.86\end{array}$ & $\begin{array}{l}0.33 \\
0.25\end{array}$ & $\begin{array}{l}1.07 \\
0.69\end{array}$ & $\begin{array}{l}0.44 \\
0.24\end{array}$ \\
\hline
\end{tabular}


Table 2

Mean Slopes and Intercepts (and Their Standard Deviations) as a Function of Presentation Duration

\begin{tabular}{lccccc}
\hline \multirow{2}{*}{$\begin{array}{c}\text { Presentation } \\
\text { Duration }\end{array}$} & \multicolumn{2}{c}{ Intercept } & & \multicolumn{2}{c}{ Slope } \\
\cline { 2 - 3 } \cline { 5 - 6 } & $M$ & $S D$ & & $M$ & $S D$ \\
\hline $500 \mathrm{msec}$ & 0.33 & 0.17 & & 0.92 & 0.17 \\
$1 \mathrm{sec}$ & 0.52 & 0.23 & & 0.84 & 0.17 \\
$2 \mathrm{sec}$ & 0.72 & 0.31 & & 0.77 & 0.16 \\
$3 \mathrm{sec}$ & 0.89 & 0.40 & & 0.74 & 0.21 \\
$5 \mathrm{sec}$ & 1.24 & 0.47 & & 0.69 & 0.23 \\
\hline
\end{tabular}

at the floor with other subjects whose discriminability was above the floor. Because the slopes should be one (i.e., equal variance) when discriminability is at the floor, this averaging artifact could produce greater slopes in the 500 msec presentation duration condition. While we attempted to deal with this problem by excluding the subjects whose intercepts were below .1 in the above analyses, we also approached it in two additional ways. First, we examined performance for all subjects whose intercepts in the 500msec presentation duration condition were below 3 to determine whether those subjects were more likely to show a decrease in slope from the 500 -msec presentation duration condition to the 2 -sec presentation duration condition than our other subjects. There was no evidence in any of our four rating conditions for such an effect. For example, in the 6-point rating condition, the effect was actually slightly smaller for the subjects whose intercept in the $500-\mathrm{msec}$ presentation duration condition was below .3. Second, we examined this issue directly in Experiment 2 by manipulating a broader range of presentation durations. By examining the change in the $z$-transformed ROC curve at greater presentation durations we attempted to verify that increases in presentation duration could produce decreases in the slope of the $z$-transformed ROC curve even when the initial presentation durations produced substantially above-chance discriminability for all subjects.

One additional question one might ask is whether our mean differences in intercept and slope (depicted visually in Figure 1) were broadly representative of the $z$ ROCs of individual subjects. They were. In fact, 108 of 128 comparisons demonstrated greater intercepts in the longer presentation duration, and 92 of 128 comparisons demonstrate smaller intercepts in the longer presentation duration condition (both $p \mathrm{~s}<.05$ ).

In Experiment 2, we examined the slope and intercept of the $z$-transformed ROC across a broad range of presentation durations from $500 \mathrm{msec}$ to $5 \mathrm{sec}$. Manipulating a broad range of presentation durations allowed us to replicate Experiment 1 and determine whether slopes can decrease in conditions in which all subjects show discriminability that is substantially above chance.

\section{EXPERIMENT 2}

\footnotetext{
Method

Subjects. The subjects were 32 students in an introductory psychology class who participated in partial fulfillment of a research requirement.
}

Design. The experiment used a one-factor within-subjects design. Presentation duration ( $500 \mathrm{msec}$ vs. $1 \mathrm{sec}$ vs. $2 \mathrm{sec}$ vs. $3 \mathrm{sec}$ vs. $5 \mathrm{sec}$ ) was manipulated within subjects.

Materials. The same materials were used as in Experiment 1. These words were divided into five sets of 128 items, with half of the items in each set presented as the study materials for one of the five within-subjects conditions. All the remaining items were presented as new items on the test, with old and new items counterbalanced across subjects. Similarly, each set of 128 items was rotated through the five within-subjects conditions across subjects. This ensured that (1) all items appeared equally often as old and new items, (2) all old items appeared equally often in each presentation duration, and (3) serial position of items was equated across presentation durations.

Procedure. The experiment consisted of the presentation of five study blocks followed by a test list that presented all the items from the study blocks with an equal number of new, distractor items. Prior to the first study list, the subjects were told they would be presented with items on a computer monitor. They were warned that some items would be presented rapidly. Following these brief instructions, 64 items were presented in one of the presentation duration conditions. Then, the 64 items were presented in another presentation duration condition, continuing until blocks of 64 items were presented in all five presentation duration conditions. Following these study lists, the subjects were asked to do 1 min of backward counting during the retention interval. After this brief retention interval, a recognition memory test presented the 320 study items and 320 distractor items in random order. For each test item, the subjects were asked to make an old-new judgment, followed by a confidence rating in which the subjects reported their confidence for all items on a 3-point scale, with 1 representing low confidence and 3 representing high confidence (i.e., the 6-rating symmetric condition of Experiment 1). Following the recognition memory test, the subjects were debriefed and excused.

\section{Results and Discussion}

The primary result of Experiment 2 was that increases in presentation duration produced decreases in the slope of the $z$-transformed ROC across a range of presentation durations. This again raises questions about the generality of prior null effects of presentation duration. Furthermore, it reinforces that the results of Experiment 1 did not arise solely as an artifact of the low levels of discriminability found in the $500-\mathrm{msec}$ condition. The mean intercepts and slopes are presented in Table 2 as a function of presentation duration. The $z$-ROCs incorporating these parameters are presented in Figure 2 as a function of presentation duration.

Visual inspection of Figure 2 demonstrates the relationship between increased presentation duration and decreased slope. One-way ANOVAs demonstrated a significant effect of presentation duration on both intercepts $\left[F(4,156)=67.09, M S_{\mathrm{e}}=0.07, p<.001\right]$ and slopes $\left[F(4,156)=13.11, M S_{\mathrm{e}}=0.03, p<.001\right]$, with intercepts increasing with presentation duration and slopes decreasing with presentation duration. Planned contrasts of performance in the $500-\mathrm{msec}$ and $2-\mathrm{sec}$ conditions replicated the results of Experiment 1, demonstrating increases in intercept and decreases in slope (both $p s<.001$ ). Planned contrasts of performance in the 2-sec and 5-sec conditions also demonstrated this pattern (both $p \mathrm{~s}<.01$ ), showing that decreases in slope can occur when all subjects show above-chance discriminability. 


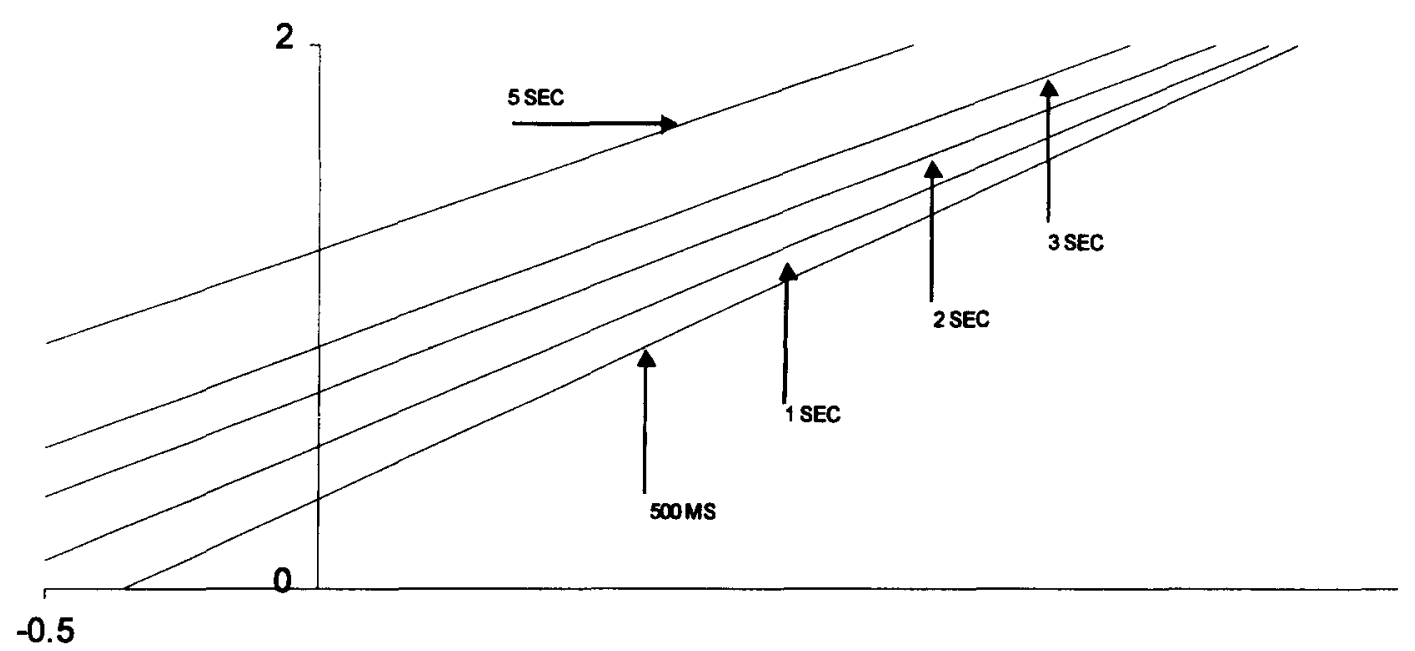

Figure 2. $z-R O C$ as a function of presentation duration.

\section{GENERAL DISCUSSION}

The results of these two experiments are straightforward. In Experiment 1 we increased presentation duration from $500 \mathrm{msec}$ to $2 \mathrm{sec}$, and the slope of the $z$-transformed ROC curve decreased across four different rating methods conditions. These results provided evidence that prior null findings of the effect of presentation duration on the slope of the $z$-transformed ROC curve were not general. In addition, these results have important implications for Yonelinas's (1994) dual-process account of the slope of the $z$-transformed ROC curve. Yonelinas argues that mixed results in prior studies can be accounted for by recognizing that the dual processes of recollection and familiarity affect the slope of the $z$-transformed ROC curve. He also argues that some variables have different effects on recollection and familiarity, producing differing effects on the slope of the $z$-transformed ROC curve. Specifically, and germane to the findings here, Yonelinas claims that manipulating presentation duration affects both recollection and familiarity. In his analysis, this operates to keep the slope of the $z$-transformed ROC curve approximately constant as presentation duration increases. In Experiment 1 , the presentation durations of $500 \mathrm{msec}$ and $2 \mathrm{sec}$ were chosen to maximize the probability that increasing presentation duration would increase both recollection and familiarity. Our finding that increasing presentation duration under these conditions reduced the slope of the $z$ transformed ROC curve raises important questions about the generality of Yonelinas's account.

In Experiment 2, we directly examined changes in the $z$-transformed ROC curve across a broad range of presentation durations. The results of Experiment 2 replicated the decreases in the slope of the $z$-transformed ROC curve from Experiment 1 across a broad range of presentation durations, including a number of durations in which discriminability was clearly above chance for all subjects.
We want to close by emphasizing where we think the present results leave us in the context of the broader literature. We think they raise important questions about the generality of prior findings that demonstrated null effects of presentation duration on the slope of the $z$-transformed ROC curve. We also think they raise important questions about the generality of prior accounts designed to explain such findings. It is important to note that we are not claiming that the present results raise questions about the reliability of the prior findings demonstrating null effects of presentation duration on the slope of the $z$-transformed ROC curve. Instead, we are attempting to point out that the pattern of results found with the variable of presentation duration is substantially more complex than we previously recognized. In some cases, an experimental manipulation of presentation duration appears to have limited or no effect on the slope of the $z$-transformed ROC curve. In other cases, as in the present study and in the studies of Glanzer et al. (1999), there appears to be a substantial effect. Thus, we think it likely that whether or not the slope of the $z$-transformed ROC curve decreases with increases in presentation duration depends on a complex set of experimental circumstances.

As further support for this point, note that the complex pattern of results occurring with the variable of presentation duration also occurs with the variable of list length. As noted earlier, Gronlund and Elam (1994, Experiment 2) and Yonelinas (1994) demonstrated that increasing list length decreased the slope of the $z$-transformed ROC, whereas Gronlund and Elam (1994, Experiment 1) and Ratcliff et al. (1994) found that increasing list length did not influence the slope of the $z$-transformed ROC curve.

We find the comparison between Gronlund and Elam's (1994) two experiments to be particularly interesting. Specifically, note that one important difference between their Experiment 1 (in which increasing list length did not affect the slope of the $z$-ROC) and their Experiment 2 (in 
which increasing list length reduced the slope of the $z$ ROC) was that many more study-test lists were presented in Experiment 1 than in Experiment 2. Interestingly, our experiments also differ from those of Ratcliff and his colleagues (e.g., Ratcliff et al., 1994) on this same dimension, with our experiments using fewer study-test lists than those of Ratcliff and his colleagues. Thus, the number of study-test lists used in experiments may have important effects on the underlying forms of the old and new distributions arising from a single study-test list, with resulting effects on the slope of the $z$-ROC. This, of course, would be broadly consistent with numerous findings demonstrating proactive interference effects in memory. In the context of the preceding remarks, the present work points the way to future studies that attempt to delineate the factors that determine whether or not the slope of $z$-transformed ROC curve will decrease with increases in presentation duration and list length. We are hopeful that the delineation of such conditions will provide boundary conditions that will constrain the development of coherent theories of the encoding and retrieval processes that underlie recognition memory.

\section{REFERENCES}

Gillund, G., \& Shiffrin, R. M. (1984). A retrieval model for both recognition and recall. Psychological Review, 19, 1-65.

GlaNZER, M., \& ADAMS, J. K. (1990). The mirror effect in recognition memory: Data and theory. Journal of Experimental Psychology: Learning, Memory, \& Cognition, 16, 5-16.

Glanzer, M., Adams, J. K., Iverson, G., \& Kim, K. (1993). The regularities of recognition memory. Psychological Review, 100, 546-567.

Glanzer, M., Kim, K., Hilford, A., \& Adams, J. K. (1999). Slope of the receiver operating characteristic in recognition memory. Journal of Experimental Psychology: Learning, Memory, \& Cognition, 25, 500513.

Green, D., \& Swets, J. (1966). Signal detection theory and psychophysics. New York: Wiley.

Gronlund, S. D., \& Elam, L. E. (1994). List-length effect: Recognition accuracy and variance of underlying distributions. Journal of Experimental Psychology: Learning, Memory, \& Cognition, 20, 1355-1369.

Hintzman, D. L. (1988). Judgments of frequency and recognition memory in a multiple-trace memory model. Psychological Review, 95, 528-551.

JACOBY, L. L. (1991). A process dissociation framework: Separating automatic from intentional uses of memory. Journal of Memory \& Language, 30, 513-541.

MURDOCK, B. B. (1982). A theory for the storage and retrieval of item and associative information. Psychological Review, 89, 609-626.

Ogilvie, J., \& Creelman, C. (1968). Maximum likelihood estimation of receiver operating characteristic curve parameters. Journal of Mathematical Psychology, 5, 377-391.

RatCliff, R., McKoon, G., \& Tindall, M. (1994). Empirical generality of data from recognition memory receiver-operating characteristic functions and implications for the global memory models. Journal of Experimental Psychology: Learning, Memory, \& Cognition, 20, 763785.

RatClifF, R., Sheu, C.-F., \& Gronlund, S. D. (1992). Testing global memory models using ROC curves. Psychological Review, 99, 518 535 .

THORNDIKE, E. L., \& LORGE, I. (1944). The teacher's word book of 30,000 words. New York: Teachers College, Columbia University.

YoNELINAS, A. P. (1994). Receiver-operating characteristics in recognition memory: Evidence for a dual-process model. Journal of Experimental Psychology: Learning, Memory, \& Cognition, 20, 13411354.

YoNELINAS, A. P. (1997). Recognition memory ROCs for item and associative information: The contribution of recollection and familiarity. Memory \& Cognition, 25, 747-763.

(Manuscript received August 21, 1998; revision accepted for publication March 7, 1999.) 